\title{
Advances in site-specific and linkage-specific ligation
}

James Tam, Giang Kien Truc Nguyen, Antony Kam, Shining Loo

School of Biological Sciences, Synzymes and Natural Products Center, Nanyang Technological University, Singapore

https://doi.org/10.17952/35EPS.2018.003

\section{Introduction}

Enzymatic peptide synthesis began, nearly 80 years ago, with the modest goal of making small peptides by reverse proteolysis using the protease chymotrypsin. Recent advances in recombinant DNA methodologies, which have made proteins and biopharmaceuticals readily available, now demanda mild and aqueous-compatible enzymatic ligation method by a true ligase for site-specific modification and precision biomanufacturing of peptide/protein-basedbiomaterials and antibody-drug conjugates.

Currently, three different groups of ligase-like enzymes or ligases with demonstrated applications have been identified. The first two groups are ligase-like enzymes which include the transpeptidase sortase A and the modified subtilisin. The third, perhaps the largest group, belongs to the naturally-occurringligases discovered in the biosynthesis of ribosomally-synthesized and post-translationally modified peptides (RiPP) which often display cyclic structures. They include TraF, PatG, PCY1, butelase 1, and POPB [1-5]. These ATP-independent and stand-alone ligases or cyclases have the advantage to perform both in vitro and in vivo ligation reaction unhamperedby an enzyme complex. With the exception of butelase 1, most RiPP-derived ligases display low ligase efficiency and will need improvements to be fully exploited.

Butelase 1, discovered in the cyclic-peptide-producing plant Clitoria ternatea, is an Asn/Asp-specific ligase. It belongs to the asparaginyl endoprotease (AEP) family, but it is virtually devoid of protease activity. Butelase 1 recognizes a C-terminal Asx-containing tripeptide motif, Asn/Asp-HisVal, to form an Asx-Xaa peptide bond where Xaa is any amino acid, either intramolecularly or intermolecularly, yielding, respectively, head-to-tail cyclic peptides or site-specific modified proteins [5-14]. 


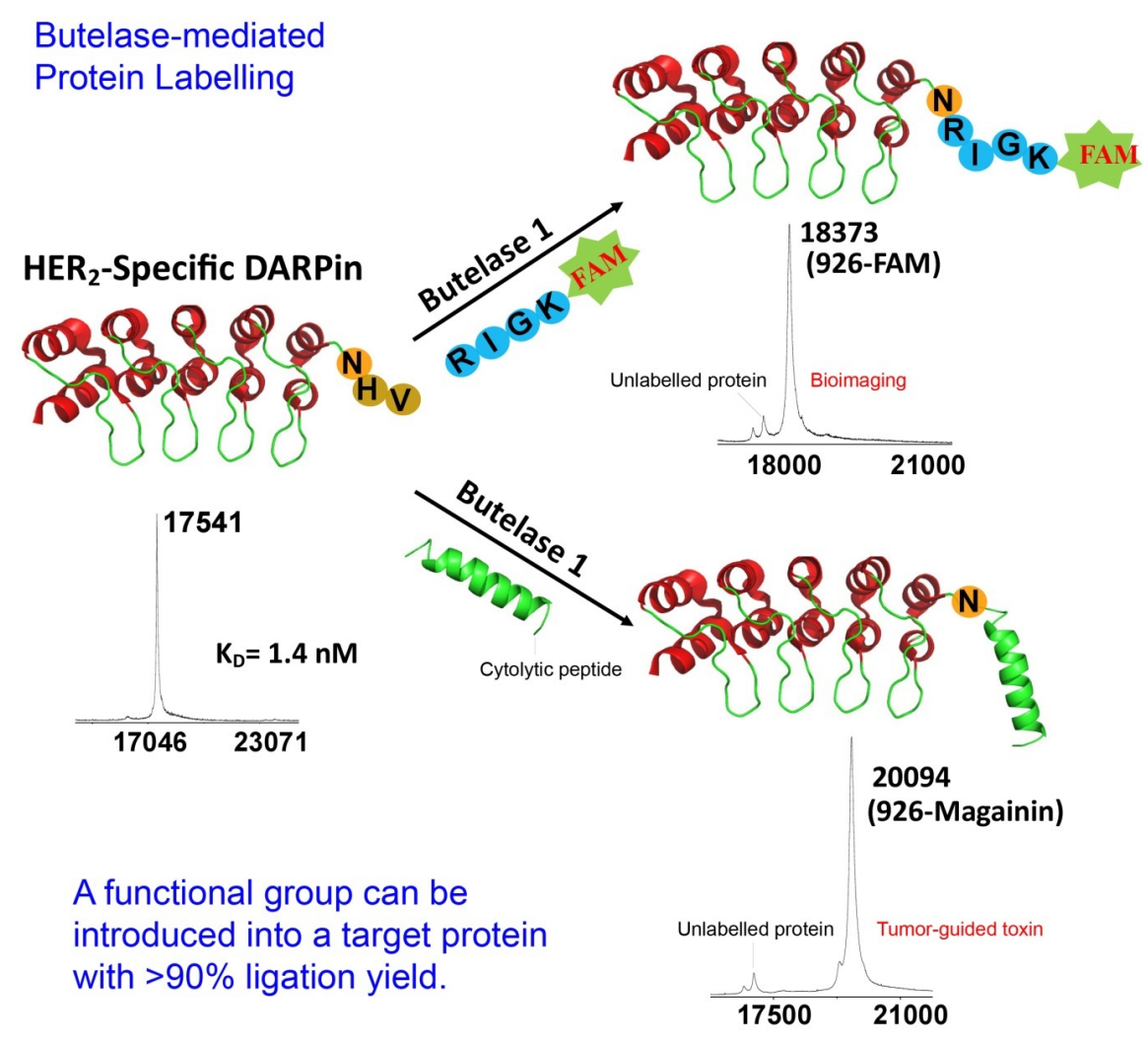

Figure 1: Butelase-mediated labeling of Her2-specific darpin 926 for bioimaging (RIGK-Fam) and targeted drug conjugates linking to a cytolytic peptide (magainin, see Figure 2).

Butelase is highly suitable for total synthesis of natural products because it does not produce any residual recognition signal sequence in the ligation product except for Asx. This advantage has been exploited for the successful synthesis of a family of high molecular weight (60-70 amin acods), hydrophobic circular peptide antimicrobials known as bacteriocins. Butelase has been shown to be compatible with orthogonal chemical ligation, or another ligase such as sortase A or subtiligase to provide variations of sequential chemoezymatic ligation or tandem ligation under one-pot conditions for modification of proteins, and antibodydrug conjugates. 


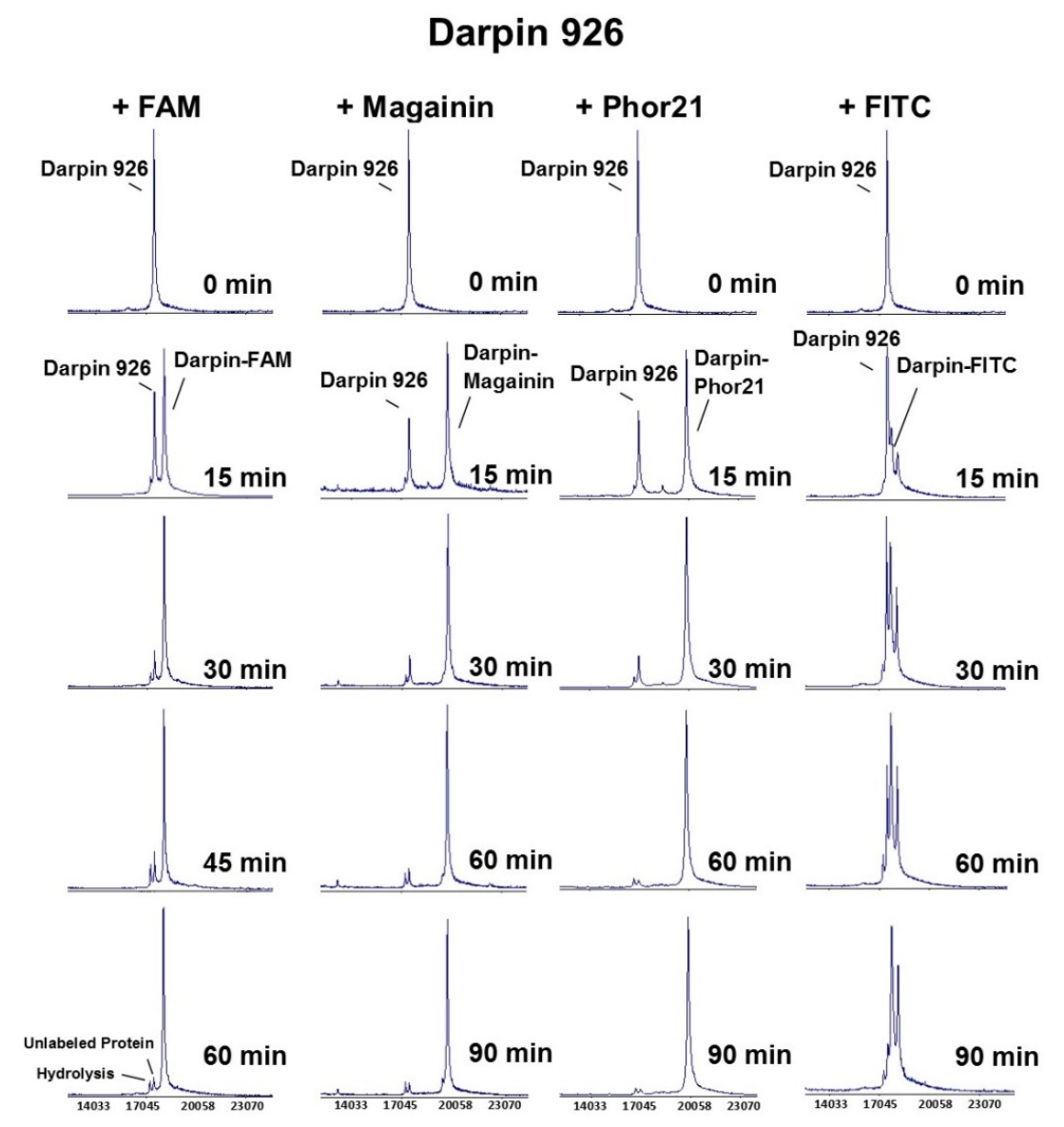

Figure 2: Time-course of butelase-mediated protein labeling of darpin 926 with FAM, Magainin, Phor21 and FITC. All reactions were performed with $100 \mathrm{nM}$ butelase 1, $25 \mu \mathrm{M}$ darpin-NHV, and $1 \mathrm{mM}$ peptide conjugates in $20 \mathrm{mM}$ sodium phosphate, $1 \mathrm{mM}$ EDTA, $40 \mu \mathrm{M} \beta$-mercaptoethanol at $42{ }^{\circ} \mathrm{C}$.

Breast cancer is the most common cancer among women, with 1.6 million new cases and a mortality rate of $>0.5$ million each year. About $20 \%$ of breast cancer are HER2-positive and treatment by monoclonal antibodies such as herceptin targeting HER2 receptor are effective. DARPins (designed ankyrin repeat proteins) represent a promising and tunable group of synthetic small non-immunoglobulin proteins. They have attracted increasing attentions as a low-cost replacement of antibodies in drug development because of their favorable molecular properties, small molecular sizes (6k vs $180 \mathrm{k}$ ), high stability and ease of production by bacterial systems. DARPin 926, a re-engineered form of darpin, was designed to achieve high binding affinity specificity towards Her2 receptor.

Here we describe the use of butelase 1 for precise prepared DARPin-conjugates targeting the breast cancer marker epidermal growth factor receptor HER2 with various payloads.

\section{Results and Discussion}

In this study, we recombinantly expressed DARPin 926 using E. coli BL21 pLysS strain with a C-terminal Asn-His-Val motif for butelase 1 recognition. Following His-tag purification and TEV cleavage, the purified protein was subjected to butelase-mediated ligation.

Cell-type-specific targeting of the butelase-mediated darpin-conjugates were shown using live-cell confocal microscopy and flow cytometry. Figures 3 and 4 demonstrated that darpin-FITC preferentially binds to Her2-positive BT474 cells, and not to Her2-negative MCF-7 cells. These results suggested that we have successfully functionalized darpin 926 to achieve Her2-positive-cell-specific targeting using butelase-mediated ligation. 


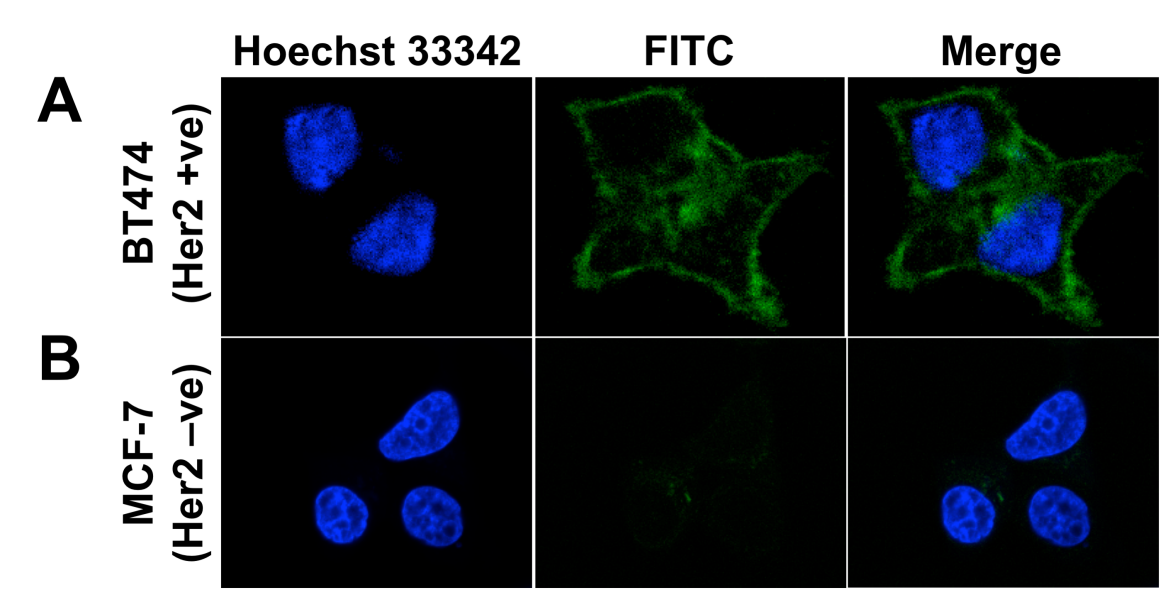

Figure 3: Confocal microscopy showed cell-type specific targeting of Darpin-FITC (green) clearly visible on the cell surface, using (A) Her2-positive (BT474), but not visible on (B) Her2-negative (MCF-7) breast cancer cells.

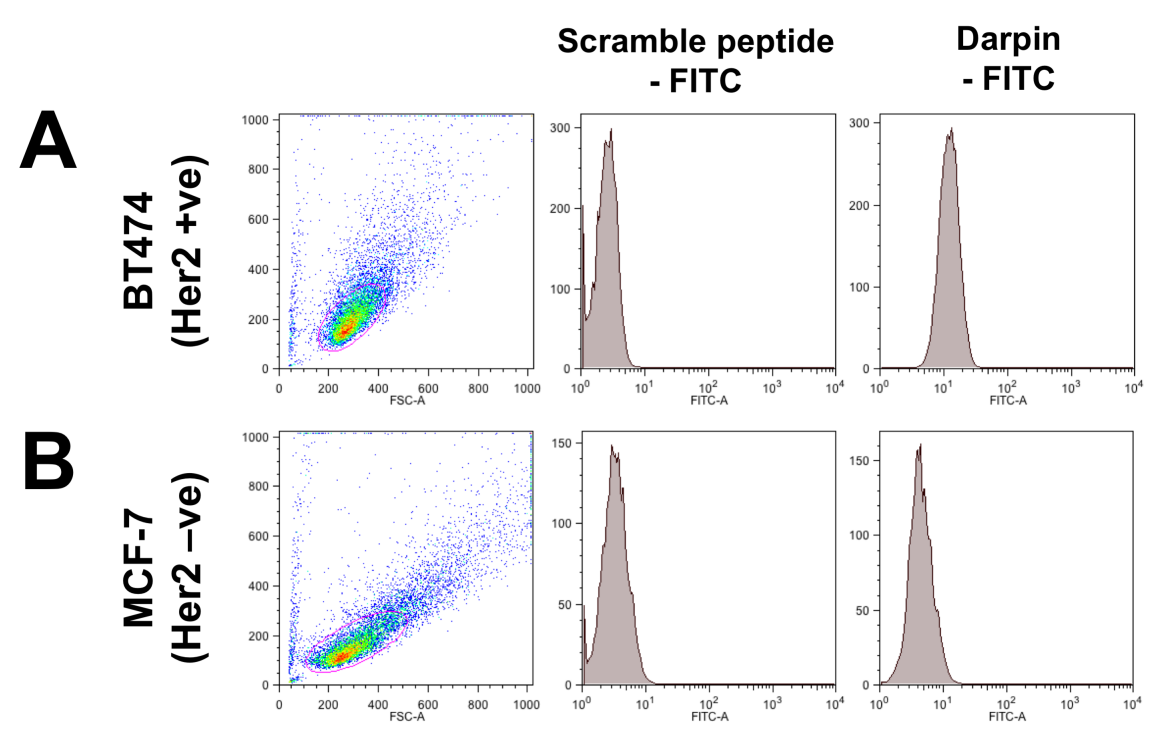

Figure 4: Flow cytometric analyses showed cell-type specific targeting of Darpin-FITC increased fluorescence intensity in (A) Her2-positive (BT474), but not in (B) Her2-negative (MCF-7) breast cancer cells.

\section{Conclusions}

In conclusion, we showed that butelase can be used for precision manufacturing of biological therapeutics with various payloads and a versatile tool to meet the demands of the coming age of precision medicine.

\section{Acknowledgments}

This research was supported by a Nanyang Technological University internal funding - 522 Synzyme and Natural Products Center (SYNC) and the AcRF Tier 3 funding (MOE2016-T3-523 1-003).

\section{References}

1. Nguyen, GKT, Wang, S, Qiu, Y, Hemu, X, Lian, Y, Tam, J P (2014). Nat Chem Biol 10: 732-8 doi: 10.1038/nchembio.1586.

2. Nguyen, GKT, Cao Y, Wang W, Liu CF, Tam JP (2015). Angew Chem Int Ed 54:15694-8 doi: 10.1002/anie.201506810. 
3. Nguyen, GKT, Kam, A, Loo, S, Jansson, AE, Pan, LX, Tam, JP (2015). J Am Chem Soc 137: 15398-401 doi: $10.1021 /$ jacs.5b11014.

4. Hemu, X, Qiu, Y, Nguyen, GKT, Tam, JP (2016). J Am Chem Soc 138: 6968-71 doi: 10.1021/jacs.6b04310 5. Nguyen, GKT, Hemu, X, Quek, JP, Tam, JP (2016). Angew Chem Int Ed 55: 12802-6 doi: 10.1002/anie.201607188.

6. Cao, Y, Nguyen, GKT, Chuah, S, Tam, JP, Liu, CF (2016). Bioconjug Chem 27: 2592-6 doi: 10.1021/acs.bioconjchem.6b00538

7. Yang, R, Wong, YH, Nguyen, GKT, Tam, JP, Lescar, J, Wu, B (2017) J Am Chem Soc 139: 5351-8 doi: 10.1021/jacs.6b12637

8. Bi, X, Yin, J, Nguyen, GKT, Rao, C, Halim, NBA, Hemu, X, Tam, JP, Liu, CF (2017). Angew Chem Int Ed 56:7822-5 doi: 10.1002/anie.201703317.

9. Bi, X, Yin, J, Hemu, X, Rao, C, Tam, JP, Liu, CF (2018). Bioconjug Chem 29: 2170-5 doi: 10.1021/acs.bioconjchem.8b00244.

10. Harmand, T, Bousbaine, D, Chan, AI, Zhang, X, Liu, DR, Tam, JP, Ploegh, HL (2018). Bioconjug Chem doi: 10.1021/acs.bioconjchem.8b00563 